\title{
Throughput Maximization of Ad-hoc Wireless Networks Using Adaptive Cooperative Diversity and Truncated ARQ *
}

\author{
Lin Dai, Member, IEEE, and Khaled B. Letaief, Fellow, IEEE \\ Center for Wireless Information Technology \\ Electrical and Electronic Engineering Department \\ The Hong Kong University of Science \& Technology \\ Clear Water Bay, HONG KONG
}

\begin{abstract}
We propose a cross-layer design which combines truncated ARQ at the link layer and cooperative diversity at the physical layer. In this scheme, both the source node and the relay nodes utilize an orthogonal space-time block code for packet retransmission. In contrast to previous cooperative diversity protocols, here cooperative diversity is invoked only if the destination node receives an erroneous packet from the source node. In addition, the relay nodes are not fixed and are selected according to the channel conditions using CRC. It will be shown that this combination of adaptive cooperative diversity and truncated ARQ can greatly improve the system throughput compared to the conventional truncated ARQ scheme and fixed cooperative diversity protocols. We further maximize the throughput by optimizing the packet length and modulation level and will show that substantial gains can be achieved by this joint optimization. Since both the packet length and modulation level are usually discrete in practice, a computationally efficient algorithm is further proposed to obtain the discrete optimal packet length and modulation level.
\end{abstract}

Keywords: Cooperative diversity, Truncated ARQ, Cross-layer design, Adaptive resource allocation, MIMO, Ad-hoc networks.

Corresponding Author:

Professor K. B. Letaief

Email: eekhaled@ee.ust.hk

Tel:/Fax: (852) 2358 7064/1485

URL: http://www.ee.ust.hk/ eekhaled

\footnotetext{
* This work is supported in part by the Hong Kong Research Grant Council under Grant No. HKUST6250/04E.
} 


\section{Introduction}

The use of multiple antennas at both the transmitter and receiver can bring significant capacity gains [1]. Unfortunately, this could be impractical in an ad-hoc wireless network, due to the size of the node or the mobile unit. In order to overcome this limitation, a new form of spatial diversity, whereby diversity gains are achieved via the cooperation of nodes, has been proposed. The main idea behind this approach, which is called cooperative diversity, is to use orthogonal relay transmission to achieve diversity gain. In particular, each node has one or several partners. The node and its partner(s) are responsible for transmitting not only their own information, but also the information of their partner(s). Therefore, a virtual antenna array is obtained through the use of the relays' antennas without complicated signal design or adding more antennas at the nodes.

Sendonaris et al proposed the idea of cooperative diversity and applied it into CDMA cellular systems. In [2-3], they presented an information-theoretic model, where two nodes cooperate by transmitting each bit over two successive bit intervals. Their results showed that node cooperation increases the sum-rate over non-cooperative transmission for ergodic fading links, given that CSI is available at the transmitter. Laneman and Wornell further extended the above work [4-5]. They thoroughly investigated the performance gain of cooperative diversity in ergodic and non-ergodic scenarios and presented several cooperative protocols, including amplify-and-forward, decode-and-forward, selection relaying and space-time-coded cooperation. Besides, Hunter et al introduced coding (RCPC codes [6-7] or turbo codes [8]) into the cooperation. This coded cooperative diversity has been shown to be able to achieve significant performance gains over the amplify-and-forward and decode-and-forward protocols [7]. Following a different approach, Stefanov and Erkip designed channel codes that can fully exploit the diversity gains of user cooperation [9]. Other important work includes cooperative regions analysis for coded cooperative protocol [10], diversity-multiplexing tradeoff analysis on cooperative protocols [11], space-time code design criteria for amplify-and-forward relay channels [12], and symbol error rate analysis for Rayleigh-fading channels with $K$ amplifying relays [13].

In most of the present cooperative protocols, no restrictions are imposed on the selection of relays. Therefore, when the channel between the source node and the relay node (s-r channel) is poor, cooperative diversity may result in even worse performance than the non-cooperative case due to 
severe error propagation. In [4], a selection relaying protocol with two nodes cooperation was proposed, where the relay forwards the source node's information only if the s-r channel fading coefficient is above a given threshold. In other words, the node is selected to be a relay only when its corresponding s-r channel is good enough. Obviously, such selective protocol can achieve better performance than the fixed ones [4]. However, it is usually not trivial to select a suitable threshold since it depends on the actual value of the channel fading coefficients. A higher threshold will reduce the possible performance gain while a lower one will allow more error propagation which also degrades the performance.

ARQ protocol at the link layer is an effective means to overcome the channel fading, where CRC is usually used for error check and retransmissions are requested if the packet is received erroneously [14-15]. In practice, the maximum number of retransmissions is usually limited so as to minimize the delay and buffer size and such variant ARQ is called truncated ARQ protocol [14]. In this paper, we propose a cross-layer design which combines truncated ARQ at the data link layer and cooperative diversity at the physical layer. We will show that through this combination, adaptive cooperative diversity gain can be achieved without any specific threshold and error propagation can be therefore avoided. In this new scheme, $Q$ idle nodes around the source node are defined as relay candidates. These nodes also receive the packet transmitted from the source node to the destination node and check the CRC results. Only the ones who detect the correct CRC are selected to be relays and involved in the possible retransmission. Specifically, if the destination node fails to detect the packet correctly, retransmission will start where both the source node and the relays utilize a suitable orthogonal space-time block code (STBC) to retransmit this packet. It can be seen that this new scheme is adaptive to the s-r channels by virtue of the CRC bits instead of some specific threshold and so no error propagation will be incurred by relaying. Besides, high efficiency can be achieved since node cooperation is adopted only when the destination node fails to detect the nodes correctly. As a result, this scheme, which is referred to as Selective Cooperative diversity with ARQ (SCA), can be expected to bring significant performance gain over the previous ARQ-only or fixed cooperative diversity schemes. Another scheme which combines truncated ARQ and fixed node cooperation is also considered in this paper. It will be shown that this scheme, which is referred to as Fixed Cooperative diversity with ARQ (FCA), requires lower complexity than SCA, while it may cause some performance loss due to error propagation. 
Throughput is defined as the data rate successfully received and regarded as a key measure of QoS for wireless systems [16]. In this paper, we focus on the throughput at the data link layer where end-to-end delivery of the packet should be guaranteed. The loss due to retransmissions of the packet is also included here. The throughput expressions of SCA and FCA are derived and compared to that of the pure truncated ARQ scheme. It will be shown that when the s-r channels are perfect, both SCA and FCA can achieve substantial gains over the truncated ARQ scheme thanks to cooperative diversity. However, with poor s-r channels, the performance of FCA will deteriorate rapidly and even become worse than the truncated ARQ scheme due to error propagation. On the other hand, SCA will always keep the highest throughput among all the schemes, which is due to its adaptability to the s-r channels.

Throughput is usually affected by many design parameters, including symbol rate, modulation level, packet length, the maximum number of retransmissions, and power level. In this paper, we further maximize the throughput by optimizing the packet length $L$ and the modulation level $b$. It is shown that substantial gains can be obtained with the joint optimization of $L$ and $b$. Besides, it is found that at low SNR we could only optimize the packet length to get an optimal throughput, whereas at high SNR, optimizing the modulation level can more effectively maximize the throughput.

In practice, the packet length $L$ and the modulation level $b$ should be discrete and under some constraints according to the specific transmission schemes. Exhaustive search over the discrete optimal $L^{*}$ and $b^{*}$ will result in prohibitive complexity. In this paper, we further present a computationally efficient algorithm, low-complexity discrete optimization algorithm (L-DOA), by which the discrete optimal values of $L^{*}$ and $b^{*}$ can be found with a rather low complexity. The comparison between the optimal throughput with continuous optimal $L^{*}$ and $b^{*}$ and that of discrete optimal $L^{*}$ and $b^{*}$ shows that in most cases, the resulting two throughput curves coincide very well, which implies that the discretization of $L^{*}$ and $b^{*}$ results in very slight throughput loss. Besides, it indicates the superior performance of L-DOA.

This paper is organized as follows. The channel model and the details of the proposed SCA and FCA are provided in Section II. Section III presents the throughput comparison of SCA, FCA and the pure truncated ARQ scheme. The throughput is further maximized by optimizing the packet length $L$ and the modulation level $b$, which is shown in Section IV. Section V presents the optimization process over discrete parameters. Finally, Section VI summarizes and concludes this paper. 


\section{Cooperative Diversity with Truncated ARQ}

In this section, we propose a new cross-layer design which combines truncated ARQ at the link layer and cooperative diversity at the physical layer.

\section{A. Scheme Description}

We consider an ad-hoc network with $K$ nodes and assume that each node is equipped with only one antenna. $Q$ idle nodes are assumed to be available as the possible relays for the source node during the packet transmission. Throughout this paper, these $Q$ nodes are referred to as relay candidates. ${ }^{1}$ Particularly, the source node transmits a data packet with a $C$-bit CRC attached. The destination node detects CRC and then sends an acknowledgement that is either positive (ACK) or negative (NACK) back to the source node. At the same time, all the $Q$ relay candidates check the CRC received from the source node and the ones who get positive results are selected to be relays. If the packet is correctly detected by the destination node (with ACK feedback), the source node continues to transmit a new data packet and the above process is repeated. Otherwise, retransmission will start. Both the source node and the relays will jointly retransmit the packet by utilizing a suitable orthogonal STBC. The retransmission continues until the packet is successfully delivered, or the number of retransmissions exceeds $N_{r}^{\max }$ which is a preset parameter indicating the maximum number of retransmissions allowed per packet. The detailed flow chart of this new scheme is shown in Fig. 1.

It can be seen that this new scheme can adapt to the s-r channels thanks to the use of CRC bits. Only the relay candidates who correctly detect the packet are selected to be relays. Adaptive cooperative diversity gain is actually achieved and error propagation can be avoided. Besides, node cooperation is adopted only when the destination node fails to detect the packet correctly. Higher efficiency can therefore be achieved compared to the previous cooperative diversity protocols. As a result, it can be expected that this proposed SCA scheme can bring significant throughput gains over those ARQ-only or fixed cooperative diversity schemes. For the sake of comparison, FCA, is also proposed in this paper, where truncated ARQ is combined with fixed node cooperation. In contrast to SCA, in FCA the relays are pre-assigned and always fixed during the whole transmission. In the retransmission, the relays send their estimates instead of the original signals. Therefore, FCA requires

\footnotetext{
${ }^{1}$ The assumption that $Q$ idle nodes are available as relay candidates can be satisfied with a proper multiuser scheduling strategy. However, a detailed study of the issues involved with the specific scheduling strategies is out of the scope of this paper.
} 
lower complexity than SCA, while it may cause some performance loss due to error propagation, which will be shown in Section III.

In SCA, the number of relays $v$ may vary in each packet transmission. In this paper, the STBC scheme is dependent on the value of $v$, i.e., we use $(v+1)$-symbol STBC in the retransmission. It should be distinguished from the space-time block coded protocol proposed in [5], where a $(Q+1)$-symbol STBC is adopted and for each cooperative transmission, $(v+1)$ columns are selected from the code matrix. It can be checked that this space-time block coded protocol can achieve the same diversity gain as ours and the complexity is rather low since each relay candidate (also the source node) is allocated a fixed orthogonal coding pattern regardless of $v$. However, it may lead to low efficiency. For instance, assume that $Q=2$ and 1 relay is selected $(v=1)$. With the space-time block coded protocol in [5], the rate $R$ is only $3 / 4 .^{2}$ Instead, the Alamouti's scheme can be adopted in our case so as to achieve the full rate $R=1$. It should be also noticed that in our scheme the relays should send a message to notify both the source node and the destination node before each retransmission. The source node then arranges the transmission order and responds to the relays. This process will result in some additional overhead compared to the conventional ARQ or previous cooperative diversity protocols. However, considering that the transmission is performed in the unit of packet (over one hundred symbols per packet, for example), this overhead can be neglected since it only requires several bits.

\section{B. Channel Model}

The communication between a source and a destination node is assumed to be over a quasi-static flat Rayleigh fading channel and facilitated by $v$ relays which are selected from $Q$ relay candidates, as shown in Fig. 2. In addition, a perfect channel knowledge is assumed to be available at the receiver side only, through the use of training sequences.

At time slots $t_{1}, \ldots, t_{L}$, the source node sends a packet $x_{t_{1}}^{s}, \ldots, x_{t_{L}}^{s}$ with transmission power $P_{t}$ per symbol, where $L$ is the packet length and $x_{t_{i}}^{s}$ is an $M$-QAM modulated symbol, $i=1, \ldots, L$. The signal received by the destination node at time slot $t_{i}, i=1, \ldots, L$, is then given by

$$
y_{t_{i}}^{d}=h_{0} x_{t_{i}}^{s}+z_{0}
$$

\footnotetext{
${ }^{2}$ Assume that the [3,4,3] STBC code given in [20] (pp. 2485, Eqn. (99)) is adopted, for instance.
} 
where the channel gain $h_{0}$ is assumed to be a complex Gaussian random variable with zero-mean and variance $\sigma_{0}^{2}$. Here $\sigma_{0}^{2}$ accounts for the effect of large-scale path loss and shadowing [5-6]. Also, $z_{0}$ represents the additive white Gaussian noise with zero-mean and variance $N_{0}$. At the $j$-th relay candidate, $j=1, \ldots, Q$, the received signal is given by

$$
y_{t_{i}}^{r_{j}}=h_{s j} x_{t_{i}}^{s}+z_{s j}
$$

where the noise $z_{s j}$ and the fading coefficient $h_{s j}$ are complex Gaussian random variables with zero-mean and variance $N_{0}$ and $\sigma_{s_{j}}^{2}$, respectively, $j=1, \ldots, Q$. In the following, the transmission of the packet $x_{t_{1}}^{s}, \ldots, x_{t_{L}}^{s}$ is referred to as direct transmission.

If the destination node fails to detect the packet correctly, retransmission will start at time slot $t_{L}+1$. Let $N_{r}$ be the number of retransmissions, then the received signal at the destination node at time slot $t_{L}+N_{r} L+i$ is given by

$$
y_{t_{L}+N_{r} L+i}^{d}=h_{0} x_{t_{L}+N_{r} L+i}^{s}+z_{0}+\sum_{j=1}^{v} h_{r j} x_{t_{L}+N_{r} L+i}^{r_{j}}+z_{r j}
$$

for $i=1, \ldots, L . x_{t_{L}+N_{r} L+i}^{s}$ and $x_{t_{L}+N_{r} L+i}^{r_{j}}$ are the space-time block coded symbols transmitted by the source node and the $j$-th relay node with the transmission power $P_{t} /(v+1)$, respectively. The additive noise $z_{r j}$ and the fading coefficient $h_{r j}$ are complex Gaussian random variables with zero-mean and variance $N_{0}$ and $\sigma_{r_{j}}^{2}$, respectively, $j=1, \ldots, v$. In this paper, it is assumed that the large scale fading of each r-d channel (the channel between the relay and the destination node) is the same as that of the s-d channel (the channel between the source node and the destination node), i.e., $\sigma_{r_{1}}^{2}=\cdots=\sigma_{r_{v}}^{2}=\sigma_{0}^{2}$.

Throughout this paper, $\gamma_{s d}$ and $\gamma_{s r}$ represent the average SNR per symbol of the direct transmission and the retransmission, respectively. $\quad p_{p d}$ and $\quad p_{p r}$ denote the packet error rate (PER) of the direct transmission and the retransmission, respectively. Similarly, $p_{s d}$ and $p_{s r}$ are the symbol error rate (SER) of the direct transmission and the retransmission, respectively.

\footnotetext{
${ }^{3}$ The difference of the large-scale fading among the r-d channels and the s-d channel can be easily compensated by power control, i.e., different transmission power levels should be allocated to each relay or the source node according to its corresponding $\sigma_{r_{j}}^{2}$ or $\sigma_{0}^{2}$. For simplicity, we assume that the large scale fading effect is the same and so equal power allocation is adopted in this paper.
} 


\section{Throughput Analysis}

In this section, the throughput of both SCA and FCA is analyzed. For the sake of comparison, the throughput expression of the pure truncated ARQ scheme is also provided. ${ }^{4}$

\section{A. Truncated ARQ scheme Throughput}

Assume that the total length of a data packet is $L$, where a $C$-bit CRC is attached and a square $M$-QAM is adopted with $b=\log _{2} M$ bit/symbol. The symbol rate $R_{s}$ is assumed to be constant and thus omitted in the following. For a point-to-point single transmission, the throughput is then given by [16]

$$
T=\frac{L-C}{L} b\left(1-p_{s d}\right)^{L / b}
$$

where $p_{s d}$ is the SER of the direct transmission.

With truncated ARQ, retransmission will start if the packet is detected erroneously and will continue until the packet is successfully delivered or the number of retransmissions $N_{r}$ exceeds $N_{r}^{\max }$. In the retransmission, the data rate will be reduced since the packet is repeated. Therefore, the throughput of the truncated ARQ scheme can be obtained as

$$
T_{A R Q}=\frac{L-C}{L} b \cdot P_{A} / \bar{N}_{A}
$$

where

$$
P_{A}=1-p_{p d}\left(p_{p r}\right)^{N_{r}^{\max }}
$$

is the packet successful rate. $\bar{N}_{A}$ is the average number of retransmissions per packet, which is given by

$$
\bar{N}_{A}=1 \cdot\left(1-p_{p d}\right)+\sum_{i=2}^{N_{r}^{\max }} i \cdot p_{p d}\left(p_{p r}\right)^{i-2}\left(1-p_{p r}\right)+\left(N_{r}^{\max }+1\right) \cdot\left(p_{p d}\left(p_{p r}\right)^{N_{r}^{\max }-1}\left(1-p_{p r}\right)+p_{p d}\left(p_{p r}\right)^{N_{r}^{\max }}\right)
$$

In (7), $p_{p d}$ and $p_{p r}$ are given by

$$
p_{p d}=1-\left(1-p_{s d}\right)^{L / b} \text { and } p_{p r}=1-\left(1-p_{s r}\right)^{L / b} \text {. }
$$

\footnotetext{
${ }^{4}$ In this section, we adopt a rather abstract system model. For example, we only consider uncoded M-QAM and the additional overhead in ARQ signaling (such as ACK and NACK) is neglected. However, the mathematical framework is general and the throughput analysis can be easily extended to the specific systems.
} 
Obviously in the truncated ARQ scheme, the SER of the retransmission $p_{s r}=p_{s d}$. Therefore, we have $p_{p r}=p_{p d}$ and (5) can then be simplified as

$$
T_{A R Q}=\frac{L-C}{L} b \cdot\left(1-p_{p d}\right) .
$$

From (9) and (4), it can be seen that the truncated ARQ scheme has exactly the same throughput as the direct transmission. Although the truncated ARQ scheme can effectively improve the reliability, it requires more transmission time. As a result, no throughput gain can be actually achieved. Nevertheless, we will show that by combining the truncated ARQ scheme and cooperative diversity, the SER of the retransmission will be improved greatly so that significant throughput gains can be obtained.

In this paper, it is assumed that the s-d channel is a flat Rayleigh fading channel. As such, the closed-form expression for the average SER of $M$-QAM is given by [17]

$$
p_{s d}=2\left(1-\frac{1}{\sqrt{2^{b}}}\right)\left(1-\sqrt{\frac{g \gamma_{s d}}{1+g \gamma_{s d}}}\right)+\left(1-\frac{1}{\sqrt{2^{b}}}\right)^{2} \cdot\left[\frac{4}{\pi} \sqrt{\frac{g \gamma_{s d}}{1+g \gamma_{s d}}} \arctan \left(\sqrt{\frac{1+g \gamma_{s d}}{g \gamma_{s d}}}\right)-1\right]
$$

where $g=\frac{3}{2\left(2^{b}-1\right)} \cdot \gamma_{s d}$ is the average SNR per symbol of the direct transmission, which is given by $\gamma_{s d}=\sigma_{0}^{2} P_{t} / N_{0}$. Therefore, by substituting (10) into (8) and (9), the throughput of the pure truncated ARQ scheme, $T_{A R Q}$, can be computed.

\section{B. Throughput of FCA}

In FCA, $v$ pre-assigned relays and the source node are used in the retransmission and both utilize a $(v+1)$-symbol orthogonal STBC to send the packet together. Therefore, the throughput of FCA can be obtained as

$$
T_{F C A}=\frac{L-C}{L} b \cdot P_{F} / \bar{N}_{F}
$$

where $P_{F}$ is the packet successful rate which can be computed by (6). $\bar{N}_{F}$ is the average number of retransmissions per packet, which is given by

$$
\bar{N}_{F}=1 \cdot\left(1-p_{p d}\right)+\frac{1}{R}\left[\sum_{i=2}^{N_{r}^{\max }} i \cdot p_{p d}\left(p_{p r}\right)^{i-2}\left(1-p_{p r}\right)+\left(N_{r}^{\max }+1\right) \cdot p_{p d}\left(p_{p r}\right)^{N_{r}^{\max }-1}\right]
$$


where $R$ is the rate of STBC (for a $k$-symbol-T-slot STBC, $R=\frac{k}{T}$ ). In this case, the average SER of the retransmission $p_{s r} \neq p_{s d}$ since multiple antenna transmission is adopted in the retransmission. In FCA, the retransmission can be regarded as $(v+1)$-transmit-1-receive STBC with $M$-QAM symbols over Rayleigh fading channels. From [18], we know that the average SER of $m$-transmit-n-receive STBC with $M$-QAM in Rayleigh fading channels is given by

$$
p_{s}=\frac{2 q \phi_{r}(g)}{\sqrt{\pi}} \frac{\Gamma(m n+1 / 2)}{\Gamma(m n+1)} \cdot{ }_{2} F_{1}\left\{m n ; \frac{1}{2} ; m n+1 ; \frac{1}{1+g \gamma_{s}}\right\}-\frac{2 q^{2}}{\pi} \frac{\phi_{r}(2 g)}{2 m n+1} F_{1}\left\{1, m n, 1 ; m n+\frac{3}{2} ; \frac{1+g \gamma_{s}}{1+2 g \gamma_{s}}, \frac{1}{2}\right\}
$$

where $\gamma_{s}$ is the average SNR per symbol, $q=1-1 / \sqrt{2^{b}}, \phi_{r}(s) \triangleq\left(1+s \gamma_{s}\right)^{-m n}$, and ${ }_{2} F_{1}(a, b ; c ; x)$ is the Gauss hypergeometric function defined as

$$
{ }_{2} F_{1}(a, b ; c ; x) \triangleq \sum_{n=0}^{\infty} \frac{(a)_{n}(b)_{n}}{(c)_{n} n !} x^{n}
$$

In the above equation, $(a)_{n}=\Gamma(a+n) / \Gamma(a)$ with $\Gamma(\cdot)$ denoting the Gamma function. Likewise, $F_{1}(a, b, b ; c ; x, y)$ is the Appell hypergeometric function defined as

$$
F_{1}\left(a, b, b^{\prime} ; c ; x, y\right) \triangleq \sum_{n=0}^{\infty} \sum_{k=0}^{\infty} \frac{(a)_{n+k}(b)_{n}\left(b^{\prime}\right)_{k}}{(c)_{n+k} n ! k !} x^{n} y^{k}
$$

Therefore, $p_{s r}$ can be obtained by substituting $m=v+1, \quad n=1$, and $\gamma_{s}=\gamma_{s r}$ into (13).

The computation of $\gamma_{s r}$ should include the effect of error propagation since in FCA the relay nodes retransmit their estimates instead of the original signals. As a result, $\gamma_{s r}$ will not be given by $\frac{\gamma_{s d}}{(v+1) R}$. Instead, the expression of $\gamma_{s r}$ of FCA is given in Theorem 1.

Theorem 1: The average SNR per symbol of the retransmission in FCA, $\gamma_{s r}$, is given by

$$
\gamma_{s r}=\frac{\gamma_{s d}}{a \gamma_{s d}+(v+1) R}
$$

where

$$
a=\frac{24(v+1)}{2^{b}-1}\left(1-2^{-b / 2}\right)\left[\left(1-\sqrt{\frac{g \gamma_{s s-r}}{1+g \gamma_{s s-r}}}\right)+\left(1-2^{-b / 2}\right) \cdot\left(\frac{4}{\pi} \sqrt{\frac{g \gamma_{s s-r}}{1+g \gamma_{s s-r}}} \arctan \left(\sqrt{\frac{1+g \gamma_{s s-r}}{g \gamma_{s s-r}}}\right)-1\right)\right] .
$$


$\gamma_{s s-r}$ is the minimum average SNR per symbol of the s-r channels and is given by $\gamma_{s s-r}=\sigma_{s_{x}}^{2} P_{t} / N_{0}$, where $\sigma_{s_{x}}^{2}=\min _{i=1, \ldots, v} \sigma_{s_{i}}^{2}$.

Proof: See the Appendix.

By combining Theorem 1 and (11-12), the throughput of FCA, $T_{F C A}$, can be obtained.

\section{Throughput of SCA}

In SCA, the number of relay nodes $v$ is not fixed. Only the candidates who detect the correct CRC results are involved in the retransmission. Therefore, the throughput expression of SCA should be written as

$$
T_{S C A}=\frac{L-C}{L} b \cdot P_{S} / \bar{N}_{S}
$$

where $P_{S}$ and $\bar{N}_{S}$ are the packet successful rate and the average number of retransmissions per packet, respectively. They are given by

$$
P_{S}=\sum_{j=0}^{Q}\left(1-p_{p d}\left(p_{p r}(j)\right)^{N_{r}^{\max }}\right) \cdot P(v=j)
$$

and

$$
\bar{N}_{S}=\sum_{j=0}^{Q} N(j) \cdot P(v=j)
$$

with $N(j)$ given by

$$
N(j)=1-p_{p d}+\frac{p_{p d}}{R(j)}\left[\sum_{i=2}^{N_{r}^{\max }} i \cdot p_{p r}(j)^{i-2}\left(1-p_{p r}(j)\right)+\left(N_{r}^{\max }+1\right) \cdot p_{p r}(j)^{N_{r}^{\max }-1}\right]
$$

for $j=0, \ldots, Q$.

In (21), $R(j)$ is the rate of a $(j+1)$-symbol STBC (let $R(0)=1)$ and $p_{p r}(j)$ is the PER of the j-relay retransmission. ${ }^{5}$ In SCA, no error propagation is introduced by relays. Therefore, with a $j$-relay retransmission $(j>0), \quad p_{s r}(j)$ can be obtained via (13) by substituting $m=j, n=1$, and $\gamma_{s}=\gamma_{s r}(j)=\frac{\gamma_{s d}}{j R(j)}$. Otherwise, $p_{s r}(j)=p_{s d}($ for $j=0)$

\footnotetext{
${ }^{5}$ Note that $p_{p r}(j)$ is now indexed by $j$ since in SCA the PER of the retransmission is dependent on the number of nodes involved in the retransmission.
} 
$P(v=j)$ is the probability that $j$ relay candidates detect the correct CRC results and is given by

$$
\begin{gathered}
P(v=0)=p_{p s-r}^{1} \cdot p_{p s-r}^{2} \cdots \cdots p_{p s-r}^{Q} \\
P(v=1)=\left(1-p_{p s-r}^{1}\right) \cdot p_{p s-r}^{2} \cdots \cdots p_{p s-r}^{Q}+p_{p s-r}^{1} \cdot\left(1-p_{p s-r}^{2}\right) \cdots \cdots p_{p s-r}^{Q}+\cdots+p_{p s-r}^{1} \cdot p_{p s-r}^{2} \cdots \cdots\left(1-p_{p s-r}^{Q}\right)
\end{gathered}
$$

where $p_{p s-r}^{i}$ is the PER of the $i$-th s-r channel, $i=1, \ldots, Q$, and we have $p_{p s-r}^{i}=1-\left(1-p_{s s-r}^{i}\right)^{L / b}$. $p_{s s-r}^{i}$ is the SER of the $i$-th s-r channel and can be computed by

$$
p_{s s-r}^{i}=2\left(1-\frac{1}{\sqrt{2^{b}}}\right)\left(1-\sqrt{\frac{g \gamma_{s s-r}^{i}}{1+g \gamma_{s s-r}^{i}}}\right)+\left(1-\frac{1}{\sqrt{2^{b}}}\right)^{2} \cdot\left[\frac{4}{\pi} \sqrt{\frac{g \gamma_{s s-r}^{i}}{1+g \gamma_{s s-r}^{i}}} \arctan \left(\sqrt{\frac{1+g \gamma_{s s-r}^{i}}{g \gamma_{s s-r}^{i}}}\right)-1\right]
$$

where $g=\frac{3}{2\left(2^{b}-1\right)}$ and $\gamma_{s s-r}^{i}$ is the average SNR per symbol of the $i$-th s-r channel which is given by $\gamma_{s s-r}^{i}=\sigma_{s_{i}}^{2} P_{t} / N_{0}, \quad i=1, \ldots, Q$.

When $\sigma_{s_{i}}^{2}=\sigma_{s}^{2}, \quad i=1, \ldots, Q$, a general expression of $P(v=j)$ can be obtained as

$$
P(v=j)=C_{Q}^{j}\left(1-p_{p s-r}\right)^{j}\left(p_{p s-r}\right)^{Q-j}
$$

where $p_{p s-r}=p_{p s-r}^{i}$, for all $i=1, \ldots, Q$, and $j=0, \ldots, Q$.

Finally, by combining (18-25), the throughput of SCA, $T_{S C A}$, can be obtained.

\section{Throughput comparison}

Assume that $Q=2$, which means that $Q=2$ idle nodes are available as the relay candidates. $C=16$ bit CRC is assumed to be adopted in each packet. Assume that the packet length $L$ is 120 and QPSK is adopted (i.e., $b=2$ ). The maximum number of retransmissions $N_{r}^{\max }$ is assumed to be 3. For simplicity, $\sigma_{s_{i}}^{2}$ is assumed to be equal to $\sigma_{s}^{2}$, for $i=1, \ldots, Q$. Therefore, we have $\gamma_{s s-r}=\gamma_{s s-r}^{i}, \quad i=1, \ldots, Q$.

Fig. 3 shows the throughput results of the pure truncated ARQ, FCA with 1 relay, FCA with 2 relays and SCA when the average SNR per symbol of the s-r channel $\gamma_{s s-r}$ is $20 \mathrm{~dB}$. The $x$-axis "SNR" is referred to the average SNR per symbol of the direct transmission $\gamma_{s d} \cdot \gamma_{s s-r}=20 \mathrm{~dB}$ indicates a rather good s-r channel. In this case, cooperative diversity gain can be fully exploited to improve the SER of the retransmission and so substantial throughput gains can be expected to be 
achieved by SCA and FCA. As Fig. 3 shows, both FCA and SCA perform much better than the truncated ARQ scheme. SCA always achieves the highest throughput, which is due to its adaptability to the s-r channels. In FCA, the effect of error propagation is rather slight thanks to the good quality of the s-r channels. Therefore, the throughput can be improved significantly compared to the truncated ARQ scheme. It should be noticed that at high SNR, FCA with 1 relay gets better performance than the 2 relay case. This is because with 3 nodes cooperation, the STBC is not full rate. ${ }^{6}$ When the SNR of the s-d channel (also the r-d channels) is high enough (which implies a good diversity gain), rate loss will significantly influence the throughput. Therefore, although in the low SNR regime FCA with 2 relays can achieve a better throughput, this throughput will become less than that of the 1 relay case when SNR is high enough.

Fig. 4 shows the case when $\gamma_{s s-r}$ decreases to $15 \mathrm{~dB}$. Here the s-r channel is not good enough and therefore the performance of FCA deteriorates rapidly due to the effect of error propagation. When SNR is high which indicates a good s-d channel, FCA even gets a worse throughput than the truncated ARQ scheme. In contrast, SCA still achieves the highest throughput among all the schemes and a significant gain can be observed.

We further consider the case of $\gamma_{s s-r}=10 \mathrm{~dB}$, which indicates an even worse s-r channel. In this case, FCA cannot work since cooperative diversity gain is overwhelmed by the effect of severe error propagation. Its SER of retransmission is always worse than that of the truncated ARQ scheme. Therefore, as Fig. 6 shows, the throughput of FCA is much lower than that of the truncated ARQ scheme. Besides, FCA with 2 relays performs even worse than the 1 relay case due to rate loss (which will result in more retransmission time). SCA again has the best performance. However, due to the bad quality of s-r channels, SCA seldom uses relays and therefore it has nearly the same throughput as the pure truncated ARQ scheme.

From the above discussion, we can conclude that SCA can always achieve a significant throughput gain irrespective of whether the s-r channel is good or not. In contrast, the performance of FCA highly depends on the quality of the s-r channel. It can improve the throughput only when the s-r channel is perfect. Otherwise, the performance will deteriorate rapidly due to error propagation and may be even worse than the pure truncated ARQ scheme. This can be more clearly seen in Fig. 5,

\footnotetext{
${ }^{6}$ In this paper, we take the [3,4,3] STBC code given in [15] (pp. 2485, Eqn. (99)). Therefore, the rate $R$ is $3 / 4$.
} 
where the average SNR per symbol of the s-d channel $\gamma_{s d}$ is fixed to be $15 \mathrm{~dB}$. Here the $x$-axis is given by $\rho=\gamma_{s s-r} / \gamma_{s d}$. It can be seen that FCA can achieve a higher throughput than the truncated ARQ scheme only when $\rho$ is larger than 2. This can give us some insights on the selection of cooperation region when fixed cooperative diversity is adopted. SCA again achieves the highest throughput and substantial gains can be observed for all the values of $\rho$.

From Fig. 5, it can be also seen that a higher $\rho$ always indicates a better performance gain of SCA or FCA over the truncated ARQ. In other words, more cooperative diversity gain can be achieved with a larger ratio of $\sigma_{s}^{2}$ to $\sigma_{0}^{2}$. Neglecting the effect of shadowing, this implies that the relays should be located close to the source node. Furthermore, it is found that the performance gain will be even more significant if fewer scatters exist between the source node and the relays. As shown in Fig. 6, with the assumption of Gaussian s-r channels, substantial gains can be achieved by both SCA and FCA although $\gamma_{s s-r}$ is only 10dB. In contrast, in Rayleigh fading s-r channels, no performance gain can be obtained by SCA and FCA with the same $\gamma_{s s-r}$. Therefore, we conclude that $Q$ relay candidates should be selected from those located around the source node so as to achieve better performance.

It should be noticed that in Gaussian s-r channels, FCA with 1 relay performs the best among all the schemes at high SNR. A closer observation shows that in this case SCA is very likely to choose $Q=2$ relays since the s-r channels are with good quality. Therefore, it has very slight performance gain over FCA with 2 relays and both of them suffer from the rate loss of 3-symbols STBC.

\section{Throughput Optimization using Adaptive Techniques}

The throughput expressions of the truncated ARQ scheme, FCA and SCA have been given by (9), (11) and (18), respectively. These expressions clearly depend on two important parameters: the packet length $L$ and the modulation level $b$. As we know, a small packet length indicates that most packets arrive without errors but at the cost of a large packet overhead. A large $L$ implies higher efficiency while the packet is more susceptible to errors which cause more retransmissions. As for the modulation level $b$, a packet with a low modulation level is more robust but may result in inefficient 
use of the channel. On the other hand, a packet with a high modulation level is more liable to error but carries more information per symbol. Therefore, an appropriate $L$ or $b$ is desired so as to improve the throughput.

In this section, we will further maximize the throughput by optimizing $L$ and $b$. Since the throughput expressions are neither concave nor convex, an analytical solution for the optimal packet length $L^{*}$ and modulation level $b^{*}$ is hard to be obtained. Therefore, we resort to simulations to observe how throughput varies with $L$ and $b$ first. In this section, we always assume that both $L$ and $b$ take continuous values.

Fig. 7 shows the selected sample when SCA is adopted with $\gamma_{s s-r}=20 \mathrm{~dB}$ and $\gamma_{s d}=15 \mathrm{~dB}$. It can be seen that the throughput plane is rather smooth, which indicates a good match between the local maximum and the global maximum. Actually, we have conducted extensive simulations of these three schemes, i.e., the truncated ARQ scheme, FCA and SCA, and found that the throughput plane is always smooth. Therefore, based on the above observation, we resort to the Method of Hooke and Jeeves [21] to obtain the optimal $L^{*}$ and $b^{*}$ as well as the maximum throughput. Fig. 8 shows the optimized throughput curves of the truncated ARQ scheme, FCA with 1 relay, FCA with 2 relays and SCA when $\gamma_{s s-r}$ is $20 \mathrm{~dB}$. It can be seen that with the joint optimization of $L$ and $b$, the throughput can be improved greatly whichever scheme is adopted. SCA again achieves the best throughput and substantial gains can be observed over all the other schemes. The throughput of FCA with 1 relay and 2 relays are also significantly improved by the joint optimization. However, in contrast to the case with a fixed $L$ of 120 and a fixed $b$ of 2, FCA obtains a lower optimal throughput than that of the truncated ARQ scheme at high SNR. A closer observation shows that in this case the optimal packet length $L^{*}$ of these schemes is around 50, which is much lower than 120. At high SNR, the communication link provided by the s-d channel is reliable enough for such a short packet. Therefore, although better diversity gain can be achieved by node cooperation, the effect of error propagation plays a more important role here. That is why the truncated ARQ scheme can outperform FCA in this case. On the other hand, this also indicates that $\rho=\gamma_{s s-r} / \gamma_{s d}$ should be large so as to assure that performance gain can be achieved by FCA. SCA always obtains the best performance regardless of the quality of s-r channels. The results with a lower $\gamma_{s s-r}$ are similar and so we omit them here. 
We take the example of the truncated ARQ scheme to further show the throughput gains brought by the joint optimization of $L$ and $b$. As Fig. 9 shows, the optimal throughput curve with both $L$ and $b$ optimized coincides with the suboptimal one with only $L$ optimized ( $b$ is fixed to be 2 ) in the low SNR regime. At high SNRs, the suboptimal throughput curve with only $b$ optimized approaches that of the optimal one. This implies that at low SNR we could only optimize $L$ to get an optimal throughput, whereas at high SNR, optimizing $b$ can more effectively optimize the throughput. Besides, we compare the optimal packet length $L^{*}$ with $b$ fixed and that with $b$ optimized. It is found that with $b$ optimized, the optimal packet length $L^{*}$ can be greatly decreased in the high SNR regime since in this case, we can use a higher modulation instead of increasing the packet length. On the other hand, the comparison of the optimal modulation level $b^{*}$ with $L$ fixed and that with $L$ optimized shows that with $L$ optimized, even higher modulation can be adopted so as to improve the throughput. We do not present the figures due to limited space.

\section{Optimization over Discrete Parameters}

In the above analysis, $L$ and $b$ are always assumed to be continuous. However, since square $M$-QAM is adopted, $b$ should actually be even, i.e. $b=2 k, k=1,2,3, \cdots$. Besides, $L$ should be larger than $C$ and be an integer multiple of $b$, i.e. $L>C$ and $L=u b$, where $u$ is an integer. For SCA and FCA, there are even more constraints on $L$ due to the adoption of STBC. For example, for FCA with 1 relay, $u$ should be an integer multiple of 2 since a 2-symbol STBC is adopted in the retransmission. For SCA with $Q=2$, $u$ should further be an integer multiple of 6 since both 2-symbol and 3-symbol STBC may be adopted.

Exhaustive search over the discrete optimal $L^{*}$ and $b^{*}$ will result in prohibitive complexity. In this section, we present a computationally efficient algorithm to find the discrete optimal $L^{*}$ and $b^{*}$. Particularly, in the new algorithm, we start searching the optimal packet length $L^{*}$ with a fixed $b=2$ and for each incremental $b$, we compute the maximum throughput by optimizing $L$. The whole process will terminate when the throughput begins to fall. This is based on the observation that the local throughput and the global throughput always perfectly match when $b$ varies and $L$ is fixed or $L$ varies and $b$ is fixed. This new algorithm shall be referred to as low-complexity discrete optimization algorithm (L-DOA) and is described below. 
ALGORITHM I

LOW-COMPLEXITY DISCRETE OPTIMIZATION ALGORITHM (L-DOA)

Let $L=u b$ where $u$ takes on different values for different schemes. $u=i, i=1,2,3, \ldots$, for the truncated ARQ scheme, $u=2 i$ for FCA with 1 relay, $u=3 i$ for FCA with 2 relays, and $u=6 i$ for SCA with $Q=2$. $T[l, b]$ represents the throughput with a packet length $l$ and a modulation level $b$. Initialization: $k=1, T^{*}=0$;

1. $b_{k}=2 k$. Find the optimal packet length $l^{*}$ under the given $b_{k}$. Let $a=\left\lfloor l^{*} / u b_{k}\right\rfloor$.

2. Let $L_{1}=a \cdot u b_{k}$ and $L_{2}=(a+1) \cdot u b_{k}$. Compare $T\left[L_{1}, b_{k}\right]$ and $T\left[L_{2}, b_{k}\right]$.

If $T\left[L_{1}, b_{k}\right] \geq T\left[L_{2}, b_{k}\right], L_{k}=L_{1}, T_{k}=T\left[L_{1}, b_{k}\right]$;

Else $L_{k}=L_{2}, T_{k}=T\left[L_{2}, b_{k}\right]$.

3. If $T_{k}>T^{*}, T^{*}=T_{k}, L^{*}=L_{k}, b^{*}=b_{k}$;

Else stop.

4. $k=k+1$. Repeat Steps1-3.

It can be seen that with L-DOA, the joint optimization of $L$ and $b$ is decoupled so that the complexity can be reduced dramatically. Table 1 lists the discrete optimal values of $L^{*}$ and $b^{*}$ for the truncated ARQ scheme, FCA with 1 relay, FCA with 2 relays and SCA with $Q=2$ under a $\gamma_{s s-r}$ of 20dB. The corresponding throughput curves are plotted in Fig. 10. For comparison, the optimal throughput curves with continuous optimal $L^{*}$ and $b^{*}$ are also shown. It can be seen that a good match between the throughput curves with continuous optimal $L^{*}$ and $b^{*}$ and that with discrete optimal $L^{*}$ and $b^{*}$ can be achieved whichever scheme is adopted, indicating that the discretization of $L^{*}$ and $b^{*}$ results in very slight throughput loss. It also indicates the superior performance of L-DOA. A closer observation shows that a slight throughput loss will be incurred in the cases of SCA with $\gamma_{s d}=15 \mathrm{~dB}$ and FCA and the truncated ARQ scheme with $\gamma_{s d}=20 \mathrm{~dB}$. Actually, this is because the optimal $b^{*}$ is around 3 in these cases. By restricting $b$ to be even, a throughput loss will be incurred. Nevertheless, in most cases, the two corresponding throughput curves perform a perfect match, which indicates the excellent performance of L-DOA. The corresponding results with the other 
values of $\gamma_{s s-r}$ are similar and so we omit them here due to limited space.

\section{Conclusions}

In this paper, we proposed a cross-layer design combining truncated ARQ at the link layer and cooperative diversity at the physical layer, which has been shown to be able to greatly improve the system throughput. The throughput expressions of the proposed SCA scheme, FCA scheme and the pure truncated ARQ scheme are derived and the comparison of these three schemes showed that the proposed SCA scheme can always achieve the highest throughput and effectively avoid error propagation. The throughput is further maximized by optimizing the packet length $L$ and the modulation level $b$ and it is found that substantial gains can be achieved by this joint optimization. Besides, since both $L$ and $b$ are usually discrete and under some constraints in practice, we proposed a low-complexity discrete optimization algorithm (L-DOA) to search the discrete optimal $L^{*}$ and $b^{*}$. It was then shown that the discretization of $L^{*}$ and $b^{*}$ results in very slight throughput loss.

\section{Appendix}

Let $\mathbf{X}$ denote an $m$-antenna-T-time-slot- $k$-symbol STBC, then it can be represented as

$$
\mathbf{X}=\left[\mathbf{A}_{1} \mathbf{s}+\mathbf{B}_{1} \mathbf{s}^{*}, \mathbf{A}_{2} \mathbf{s}+\mathbf{B}_{2} \mathbf{s}^{*}, \ldots, \mathbf{A}_{\mathrm{T}} \mathbf{s}+\mathbf{B}_{\mathrm{T}} \mathbf{s}^{*}\right]
$$

where $\mathbf{s}$ is a $k \times 1$ complex variable vector and $\mathbf{A}_{\mathbf{i}}, \mathbf{B}_{\mathbf{i}}$ are constant coefficient matrices in $\mathcal{R}^{m \times k}$. Assume $n$ antennas at the receiver side. Then, the $n \times T$ receive signal matrix $\mathbf{Y}$ can be written as

$$
\mathbf{Y}=\sqrt{\frac{S N R}{m}} \mathbf{H X}+\mathbf{N}
$$

where $S N R=\sigma_{0}^{2} P_{t} / N_{0}, \mathbf{H}$ and $\mathbf{N}$ are the $n \times m$ channel gain matrix and $n \times T$ noise matrix, respectively. All $h_{i j}, i=1, \ldots, n, \quad j=1, \ldots, m$ and $n_{i j}, \quad i=1, \ldots, n, \quad j=1, \ldots, T$ are i.i.d. complex-valued Gaussian random variables with zero-mean and unit variance. After a series of linear transformation, we have

$$
\mathbf{r}=\sqrt{\frac{S N R}{m}} \mathcal{H} \cdot \mathbf{s}+\mathbf{z}
$$


where

$$
\mathcal{H}=\left[\begin{array}{c}
\mathbf{H} \mathbf{A}_{1}+\mathbf{H}^{*} \mathbf{B}_{1}^{*} \\
\vdots \\
\mathbf{H} \mathbf{A}_{T}+\mathbf{H}^{*} \mathbf{B}_{T}^{*}
\end{array}\right]
$$

From the property of $\left\{\mathbf{A}_{j}\right\}_{j=1, \ldots, T}$ and $\left\{\mathbf{B}_{j}\right\}_{j=1, \ldots, T}$, we have

$$
\mathcal{H}^{+} \cdot \mathcal{H}=\operatorname{trace}\left(\mathbf{H}^{+} \mathbf{H}\right) \cdot \mathbf{I}_{k}
$$

Therefore,

$$
\tilde{\mathbf{r}}=\sqrt{\frac{S N R}{m}} \alpha \cdot \mathbf{s}+\tilde{\mathbf{z}}
$$

where $\tilde{z}_{i} \sim \mathcal{N}_{c}(0, \alpha), \quad i=1, \ldots, k$, and $\alpha=\operatorname{trace}\left(\mathbf{H}^{+} \mathbf{H}\right)$. Obviously, $\alpha$ is a central chi-square distributed variable with $2 m n$ degrees of freedom. From (31), it can be seen that orthogonal space-time block decoding transforms a MIMO channel into multiple equivalent SISO channels. Therefore, s can be easily detected using a one-dimension MLD.

In FCA, $v$ relay nodes and the source node use a $v+1$ symbol STBC to send the signals together. Therefore, here we have $m=k=v+1$ and $n=1$. Note that in FCA, the relay nodes are pre-assigned and CRC will not be checked to assure a reliable information forwarding. As a result, the estimates $\hat{\mathbf{s}}$ instead of the original signal vector $\mathbf{s}$ is transmitted, where $\hat{\mathbf{s}}=\left[s_{0}, \hat{s}_{1}, \ldots, \hat{s}_{v}\right]$, and $\hat{s}_{i}$ is the estimate of relay node $i, \quad i=1, \ldots, v$. Then, (31) is modified to be

$$
\tilde{\mathbf{r}}=\mu \alpha \cdot \mathbf{s}+\mu \alpha \cdot\left[\begin{array}{c}
0 \\
\hat{s}_{1}-s_{1} \\
\vdots \\
\hat{s}_{v}-s_{v}
\end{array}\right]+\tilde{\mathbf{z}}
$$

where $\mu=\sqrt{\gamma_{s d} /(v+1)}$ and $\alpha$ is a central chi-square distributed variable with $2(v+1)$ degrees of freedom. Let $\widehat{z}_{i}=\mu \alpha\left(\hat{s}_{i}-s_{i}\right)+\tilde{z}_{i+1}$, where $i=1, \ldots, v$. Here the new equivalent noise $\hat{\mathbf{z}}$ includes both the additive white noise $\tilde{\mathbf{z}}$ and the effect of error estimates. It can be further obtained that for $i=1, \ldots, v$,

$$
E\left(\widehat{z}_{i}\right)=0 \text {, and } \operatorname{var}\left(\widehat{z}_{i}\right)=\operatorname{var}\left[\mu \alpha\left(\hat{s}_{i}-s_{i}\right)\right]+\alpha .
$$

From [18], we know that for an $m$-antenna-T-time-slot- $k$-symbol STBC transmission, the 
instantaneous SNR per symbol $\tilde{\gamma}_{s}$ of the equivalent SISO model is given by

$$
\tilde{\gamma}_{s}=\alpha P /\left(m R N_{0}\right)
$$

where $R=k / T$ is the rate of STBC, $P$ is the total transmission power, and $N_{0}$ is the noise variance. Therefore, by combining (32) and (33), the instantaneous SNR per symbol of the retransmission, $\tilde{\gamma}_{s r}$, should be given by ${ }^{7}$

$$
\tilde{\gamma}_{s r}=\frac{\mu^{2} \alpha}{R \cdot\left[\mu^{2} \alpha \cdot \max _{i=1, \ldots, v}\left[\operatorname{var}\left(\hat{s}_{i}-s_{i}\right)\right]+1\right]}
$$

Let $D\left(s_{i}-\hat{s}_{i}\right)$ denote the distance between $s_{i}$ and $\hat{s}_{i}$, then we have

$$
\operatorname{var}\left(\hat{s}_{i}-s_{i}\right)=D\left(s_{i}-\hat{s}_{i}\right) \cdot P\left(s_{i} \rightarrow \hat{s}_{i}\right)
$$

Here we assume that errors always happen between two neighbors. Therefore, the minimum distance $D_{\text {min }}\left(s_{i}-\hat{s}_{i}\right)$ is used instead of $D\left(s_{i}-\hat{s}_{i}\right)$. Let $E_{s}$ denote the average energy of the constellation. From [19], it follows that for M-QAM symbols

$$
D_{\min }\left(s_{i}-\hat{s}_{i}\right)=\frac{6 E_{s}}{2^{b}-1} .
$$

With the total transmission power $(v+1), E_{s}$ is given by [18]

$$
E_{s}=\frac{1}{R} \text {. }
$$

Besides, we have assumed that the s-r channel is flat Rayleigh fading. According to [19],

$$
P\left(s_{i} \rightarrow \hat{s}_{i}\right)=2\left(1-\frac{1}{\sqrt{2^{b}}}\right)\left(1-\sqrt{\frac{g \gamma_{s s-r}^{i}}{1+g \gamma_{s s-r}^{i}}}\right)+\left(1-\frac{1}{\sqrt{2^{b}}}\right)^{2} \cdot\left[\frac{4}{\pi} \sqrt{\frac{g \gamma_{s s-r}^{i}}{1+g \gamma_{s s-r}^{i}}} \arctan \left(\sqrt{\frac{1+g \gamma_{s s-r}^{i}}{g \gamma_{s s-r}^{i}}}\right)-1\right]
$$

where $\gamma_{s s-r}^{i}$ is the average SNR per symbol of the $i$-th s-r channel and is given by $\gamma_{s s-r}^{i}=\sigma_{s_{i}}^{2} P_{t} / N_{0}$. Let $\gamma_{s s-r}=\min _{i=1, \ldots, v} \gamma_{s s-r}^{i}$, then by substituting (36-39) into (35), we have

$$
\tilde{\gamma}_{s r}=\frac{\alpha \gamma_{s d}}{\frac{6}{2^{b}-1} w \gamma_{s d}+(v+1) R}
$$

where

\footnotetext{
${ }^{7}$ Considering that the entire performance is largely dependent on the worst stream, here $\tilde{\gamma}_{s r}$ is actually the instantaneous SNR of the worst stream, i.e., $\tilde{\gamma}_{s r}=\min _{i=1, \ldots, v} \tilde{\gamma}_{s r}^{i}$, where $\tilde{\gamma}_{s r}^{i}$ is the instantaneous SNR of the $i$-th stream.
} 


$$
w=2 \alpha\left(1-\frac{1}{\sqrt{2^{b}}}\right)\left(1-\sqrt{\frac{g \gamma_{s s-r}}{1+g \gamma_{s s-r}}}\right)+\left(1-\frac{1}{\sqrt{2^{b}}}\right)^{2} \cdot\left[\frac{4}{\pi} \sqrt{\frac{g \gamma_{s s-r}}{1+g \gamma_{s s-r}}} \arctan \left(\sqrt{\frac{1+g \gamma_{s s-r}}{g \gamma_{s s-r}}}\right)-1\right] .
$$

It can be easily checked that with a high $\gamma_{s s-r}, \sqrt{\operatorname{var}(w)} / E(w) \rightarrow 0$. This implies that the fluctuation around the mean of $w$ can be neglected. Therefore, $w$ can be replaced by $E(w)$ and $\tilde{\gamma}_{s r}$ can be further written as

$$
\tilde{\gamma}_{s r}=\frac{\alpha \gamma_{s d}}{a \gamma_{s d}+(v+1) R}
$$

where $a$ is given by (17). Finally, from [18] the average SNR per symbol of the retransmission $\gamma_{s r}$ can be obtained, as shown in (16).

\section{REFERENCES}

[1] R. D. Murch and K. B. Letaief, “Antenna systems for broadband wireless access,” IEEE Communications Magazine, Vol. 40, No. 4, pp. 76-83, April 2002.

[2] A. Sendonaris, E. Erkip and B. Aazhang, “User cooperation diversity - Part I: system description,” IEEE Trans. Commun., vol. 51, no. 11, pp. 1927-1938, Nov. 2003.

[3] A. Sendonaris, E. Erkip and B. Aazhang, "User cooperation diversity - Part II: implementation aspects and performance analysis,” IEEE Trans. Commun., vol. 51, no. 11, pp. 1939-1948, Nov. 2003.

[4] J. N. Laneman, G. W. Wornell, and D. N. C. Tse, "Cooperative diversity in wireless networks: efficient protocols and outage behavior,” IEEE Trans. Inf. Theory, vol. 50, no. 12, pp. 3062-3080, Dec. 2004.

[5] J. N. Laneman, G. W. Wornell, and D. N. C. Tse, "Distributed space-time-coded protocols for exploiting cooperative diversity in wireless networks,” IEEE Trans. Inf. Theory, vol. 49, no. 10, pp. 2415-2425, Oct. 2003.

[6] T. E. Hunter and A. Nostratinia, “Cooperation diversity through coding,” in Proc. ISIT'02, pp. 220, Lausanne, Switzerland, July 2002.

[7] T. E. Hunter and A. Nostratinia, “Performance analysis of coded cooperation diversity," in Proc. ICC'03, pp. 2688-2692, May 2003.

[8] M. Janani, A. Hedayat, T. E. Hunter and A. Nostratinia, "Coded cooperation in wireless communications: space-time transmission and iterative decoding,” IEEE Trans. Signal Processing, vol. 52, no. 2, pp. 362-371, Feb. 2004.

[9] A. Stefanov and E. Erkip, “Cooperative coding for wireless networks,” IEEE Trans. Commun., vol. 52, no. 9, pp. 1470-1476, Sep. 2004.

[10] Z. Lin, E. Erkip, and A. Stefanov, "Cooperative regions for coded cooperative systems," in Proc. Globecom’04, pp. 21-25, Dallas, TX, Nov. - Dec. 2004.

[11] N. Prasad, and M. K. Varanasi, "Diversity and multiplexing tradeoff bounds for cooperative diversity protocols,” in Proc. ISIT’04, pp. 268, July 2004.

[12] R. U. Nabar and H. Bölcskei, “Space-time signal design for fading relay channels,” in Proc. Globecom'03, 
pp. 1952-1956, San Francisco, Nov. 2003.

[13] P. A. Anghel and M. Kaveh, "Exact symbol error probability of a cooperative network in a Rayleigh-fading environment,” IEEE Trans. Wireless Commun., vol. 3, no. 5, pp. 1416-1421, Sep. 2004.

[14] E. Malkamaki and H. Leib, "Performance of truncated type-II hybrid ARQ schemes with noisy feedback over block fading channels,” IEEE Trans. Commun., vol. 48, no. 9, pp. 1477-1487, Sep. 2000.

[15] Q. Liu, S. Zhou, and G. B. Giannakis, "Cross-layer combining of adaptive modulation and coding with truncated ARQ over wireless links,” IEEE Trans. Wireless Commun., vo. 3, no. 5, pp. 1746-1755, Sep. 2004.

[16] R. J. Lavery, “Throughput optimization for wireless data transmission,” M. S. Thesis, Polytechnic University, June 2001, http://eeweb.poly.edu/dgoodman/thesis.html .

[17] M.-S. Alouini and A. Goldsmith, "A unified approach for calculating error rates of linearly modulated signals over generalized fading channels,” in Proc. ICC’98, pp. 459-464, June 1998.

[18] H. Shin and J. H. Lee, "Exact symbol error probability of orthogonal space-time block codes," in Proc. Globecom’02, pp. 1197-1201, Nov. 2002.

[19] F. Xiong, Digital Modulation Techniques: Artech House, 2000.

[20] M. S. Bazaraa, Nonlinear Programming: Theory and Algorithms: Wiley, 1993.

[21] X. Liang, “Orthogonal designs with maximum rates,” IEEE Trans. Inf. Theory, vol. 49, no. 10, pp. 2468-2503, Oct. 2003. 
TABLE I

OPTIMAL $L^{*}$ AND $b^{*}$ SEARCHED BY L-DOA FOR THE TRUNCATED ARQ, FCA AND SCA $\left(\gamma_{s s-r}=15 \mathrm{~dB}\right)$

\begin{tabular}{|c|c|c|c|c|c|c|}
\hline \multicolumn{2}{|c|}{ SNR $\left(\gamma_{s d}\right)$} & $0 \mathrm{~dB}$ & $5 \mathrm{~dB}$ & $10 \mathrm{~dB}$ & $15 \mathrm{~dB}$ & $20 \mathrm{~dB}$ \\
\hline $\begin{array}{c}\text { Truncated } \\
\text { ARQ Scheme } \\
(u=i)\end{array}$ & $L^{*}$ & 20 & 22 & 30 & 42 & 40 \\
\cline { 2 - 7 } & $b^{*}$ & 2 & 2 & 2 & 2 & 4 \\
\hline $\begin{array}{c}\text { FCA with } \\
v=1 \text { relay } \\
(u=2 i)\end{array}$ & $L^{*}$ & 20 & 24 & 32 & 48 & 68 \\
\cline { 2 - 7 } & $b^{*}$ & 2 & 2 & 2 & 2 & 2 \\
\hline $\begin{array}{c}\text { FCA with } \\
v=2 \text { relay } \\
(u=3 i)\end{array}$ & $L^{*}$ & 24 & 30 & 42 & 54 & 66 \\
\cline { 2 - 7 } & $b^{*}$ & 2 & 2 & 2 & 2 & 2 \\
\hline $\begin{array}{c}\text { SCA with } \\
Q=2\end{array}$ & $L^{*}$ & 24 & 36 & 48 & 60 & 48 \\
\cline { 2 - 7 }$(u=6 i)$ & $b^{*}$ & 2 & 2 & 2 & 2 & 4 \\
\hline
\end{tabular}




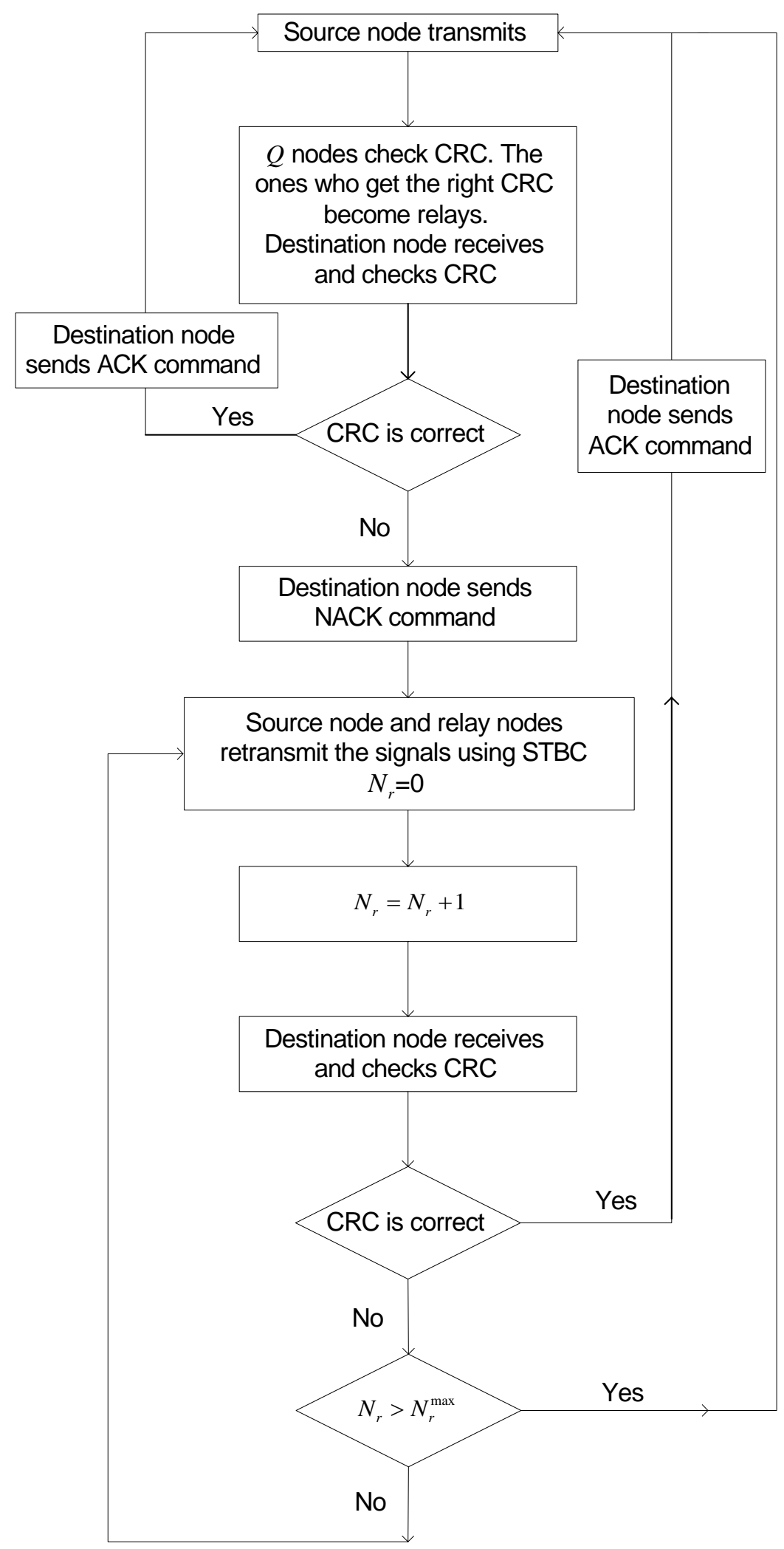

Fig. 1: Flow chart of SCA 


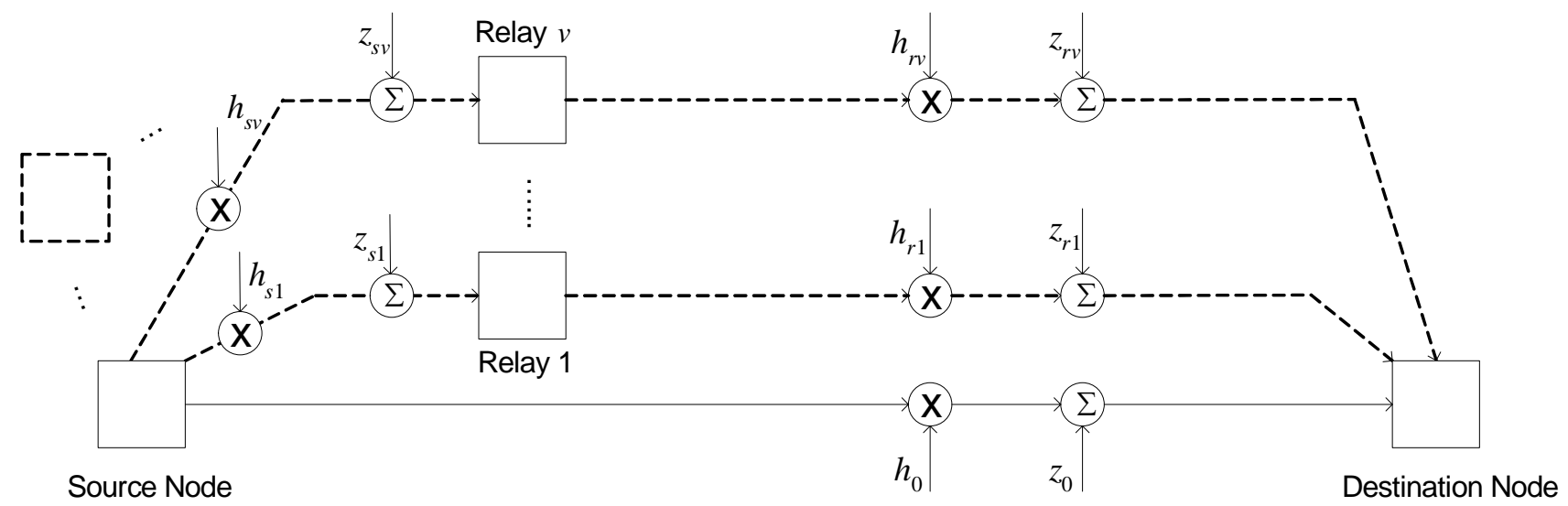

Fig. 2: System model. $Q$ idle nodes are assumed to be available as the relay candidates.

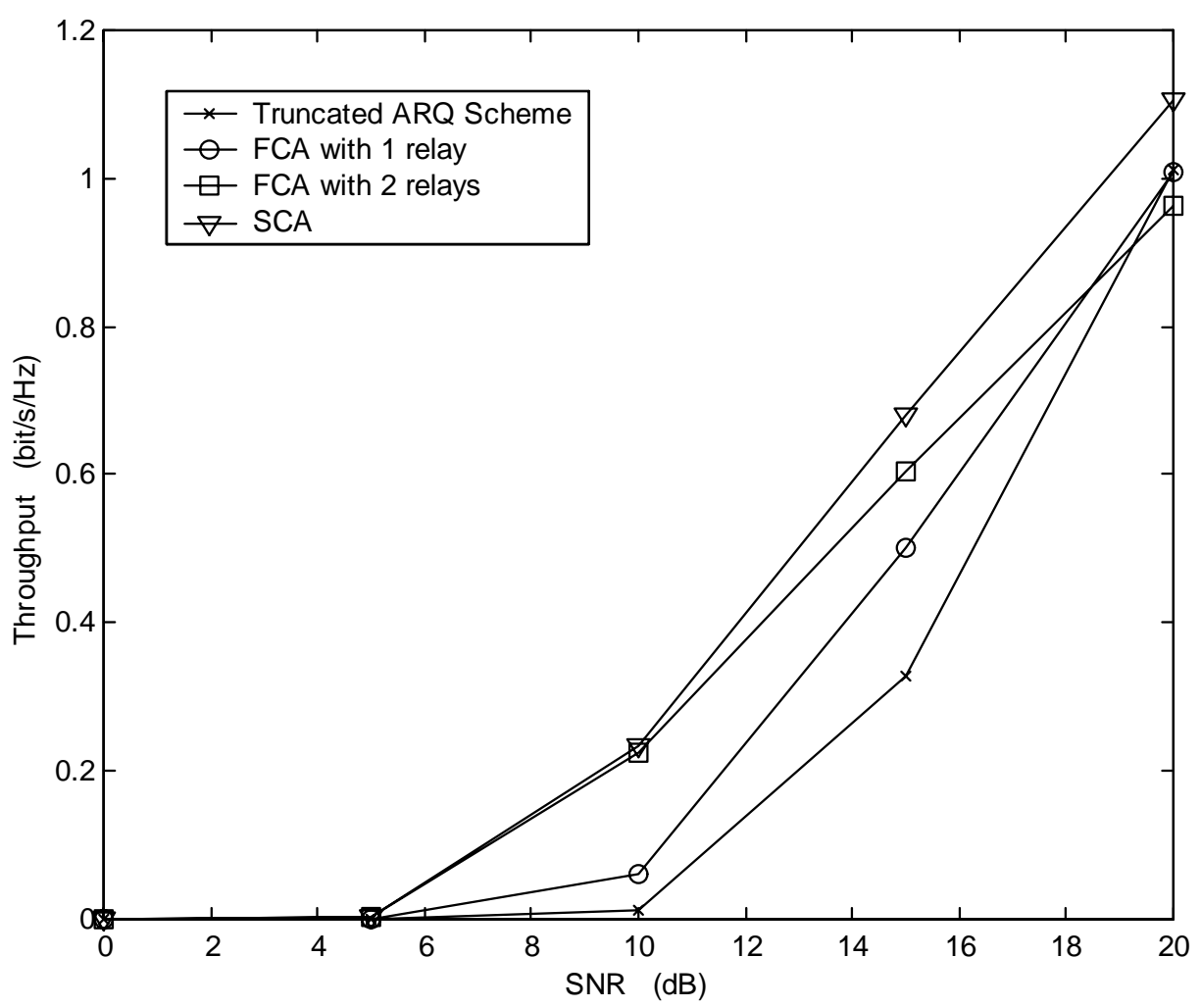

Fig. 3: Throughput of the truncated ARQ scheme, FCA with 1 relay, FCA with 2 relays and SCA when $\gamma_{s s-r}=20 \mathrm{~dB}$ with $L=120$ and $b=2$. 


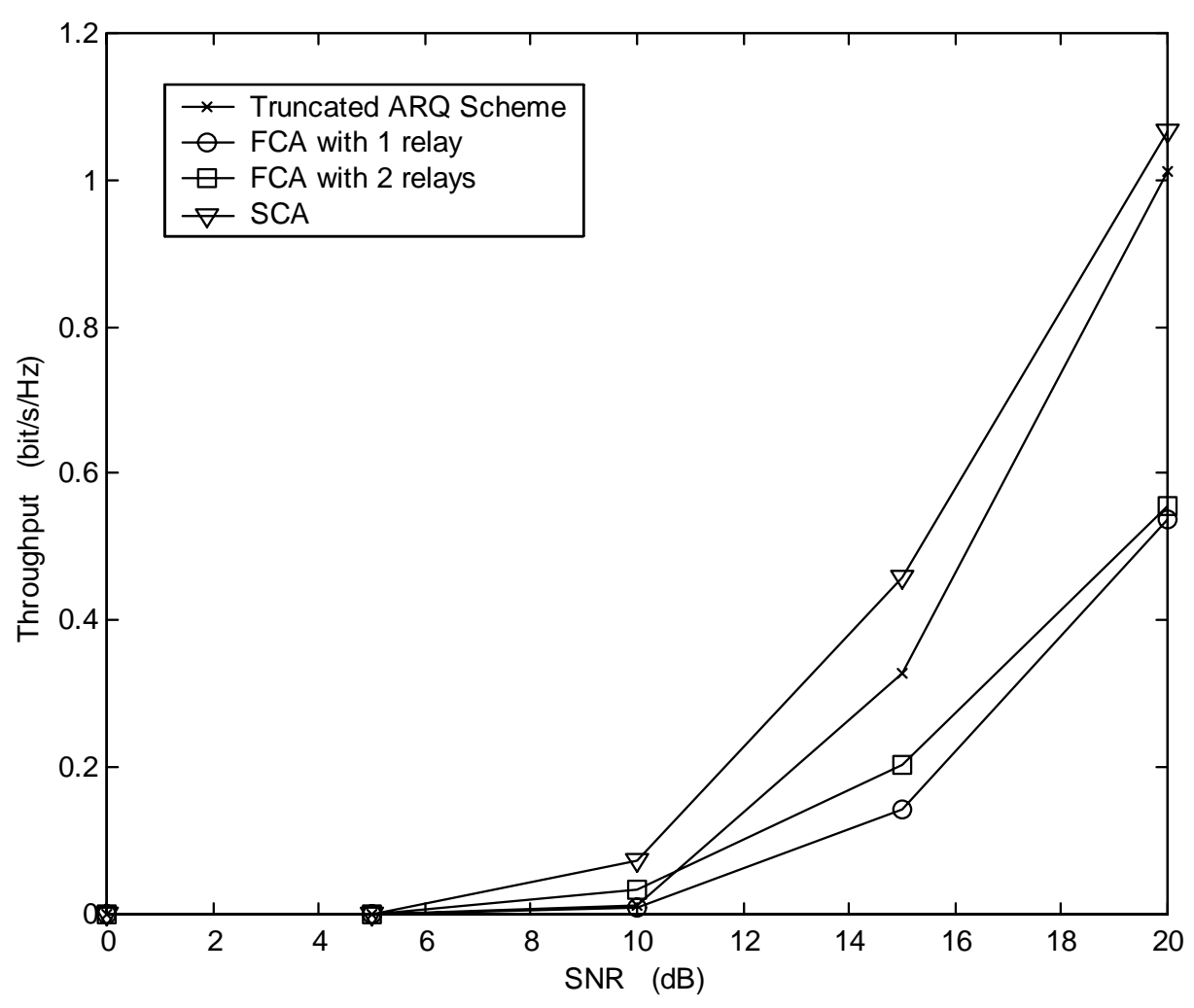

Fig. 4: Throughput of the truncated ARQ scheme, FCA with 1 relay, FCA with 2 relays and SCA when $\gamma_{s s-r}=15 \mathrm{~dB}$ with $L=120$ and $b=2$.

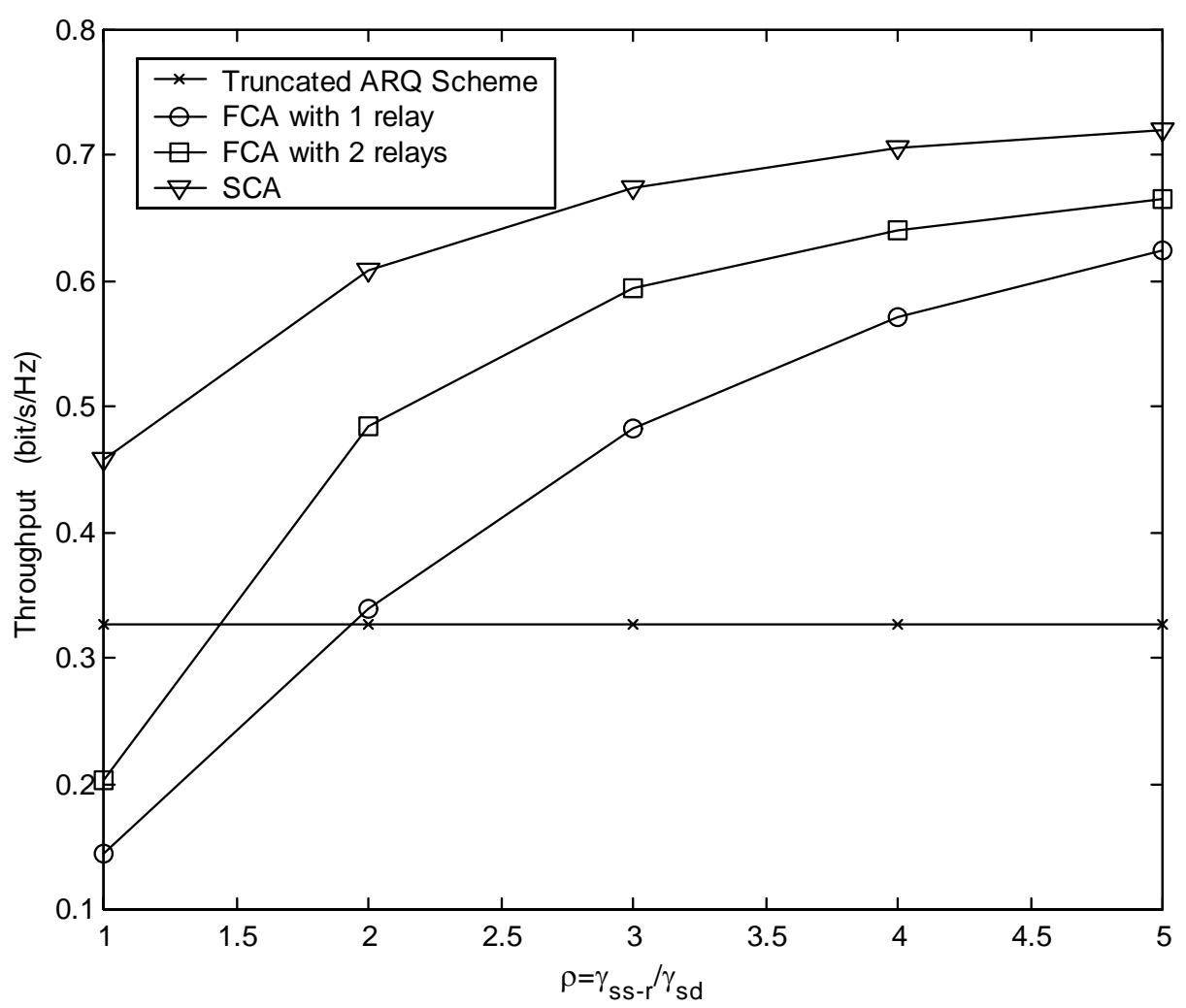

Fig. 5: Throughput of the truncated ARQ scheme, FCA with 1 relay, FCA with 2 relays and SCA when $\gamma_{s d}=15 d B$ with $L=120$ and $b=2$. 


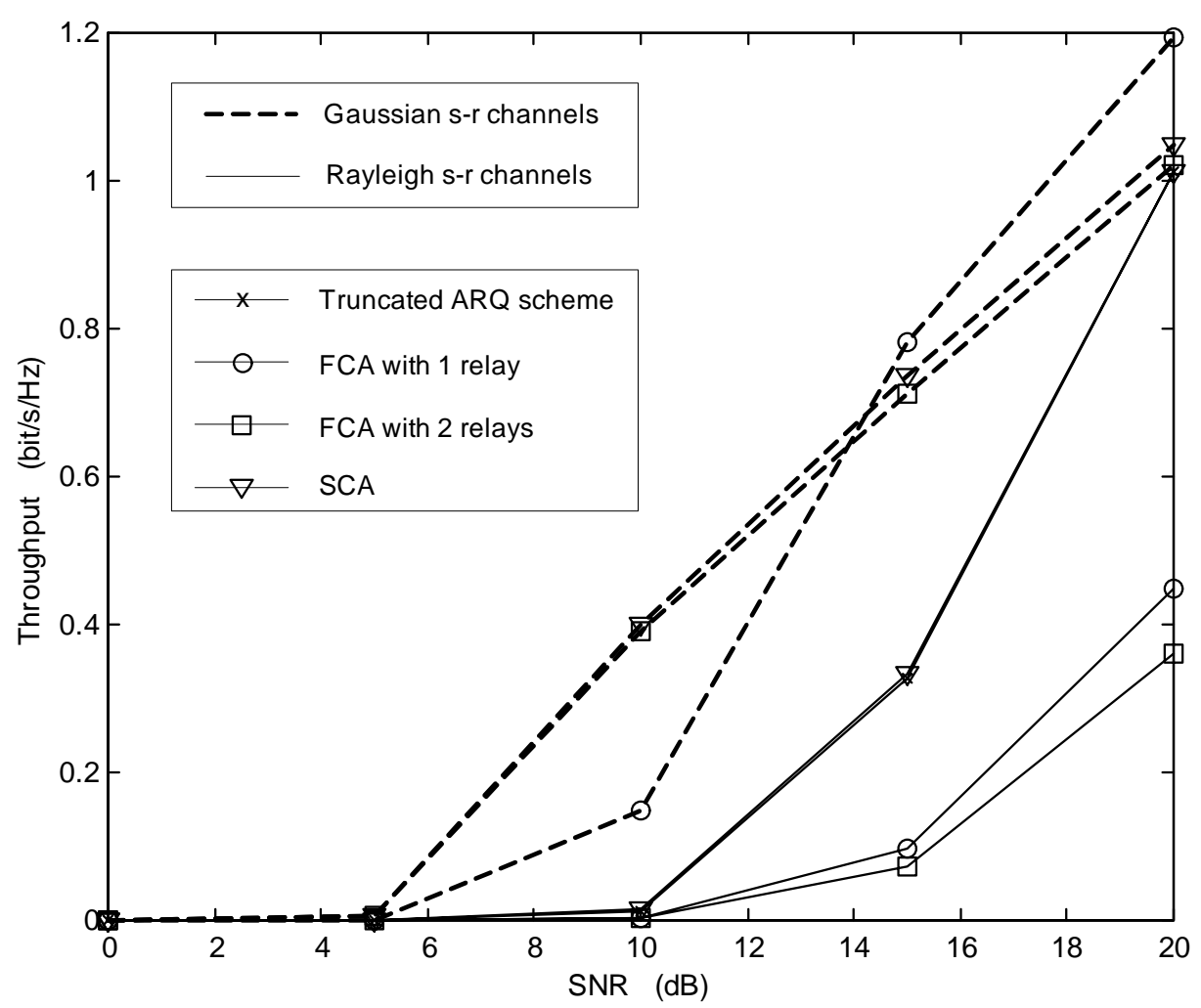

Fig. 6: Throughput of the truncated ARQ scheme, FCA with 1 relay, FCA with 2 relays and SCA with Gaussian s-r channels and Rayleigh s-r channels when $\gamma_{s s-r}=10 \mathrm{~dB}$ with $L=120$ and $b=2$.

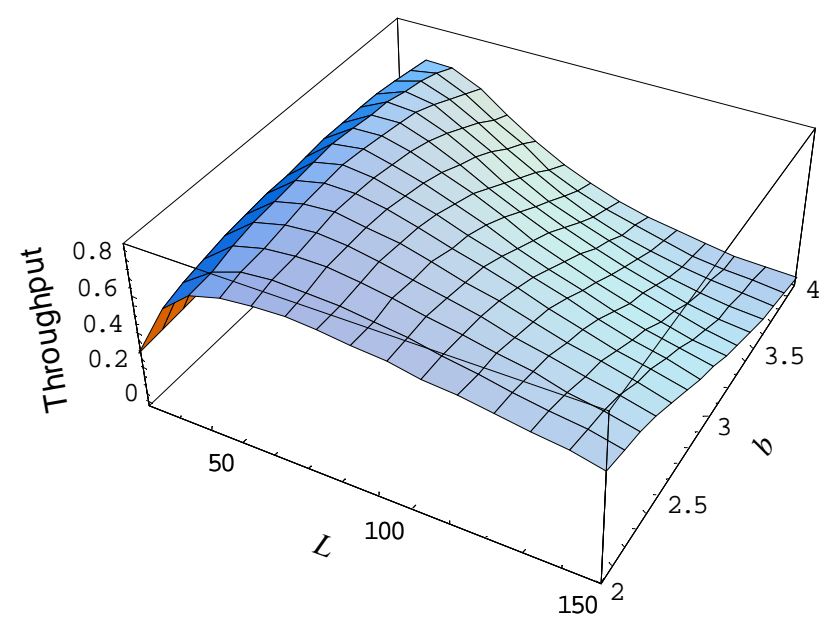

Fig. 7: Throughput plane of SCA versus $L$ and $b$ with $\gamma_{s d}=15 \mathrm{~dB}$ and $\gamma_{s s-r}=20 \mathrm{~dB}$ 


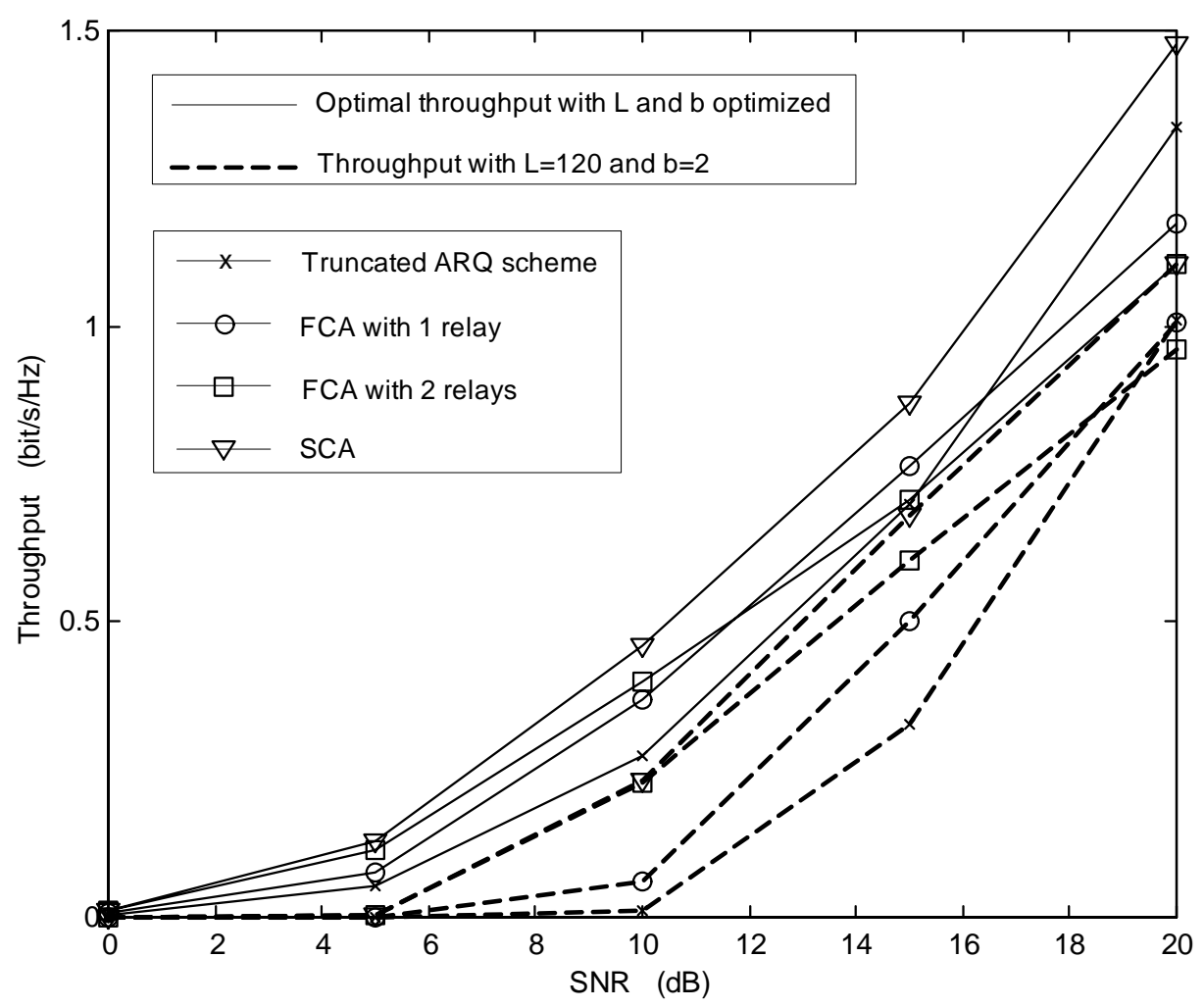

Fig. 8: Optimal throughput vs. throughput with fixed $L$ and $b$ of the truncated ARQ scheme, FCA with 1 relay, FCA with 2 relays and SCA when $\gamma_{s s-r}=20 \mathrm{~dB}$

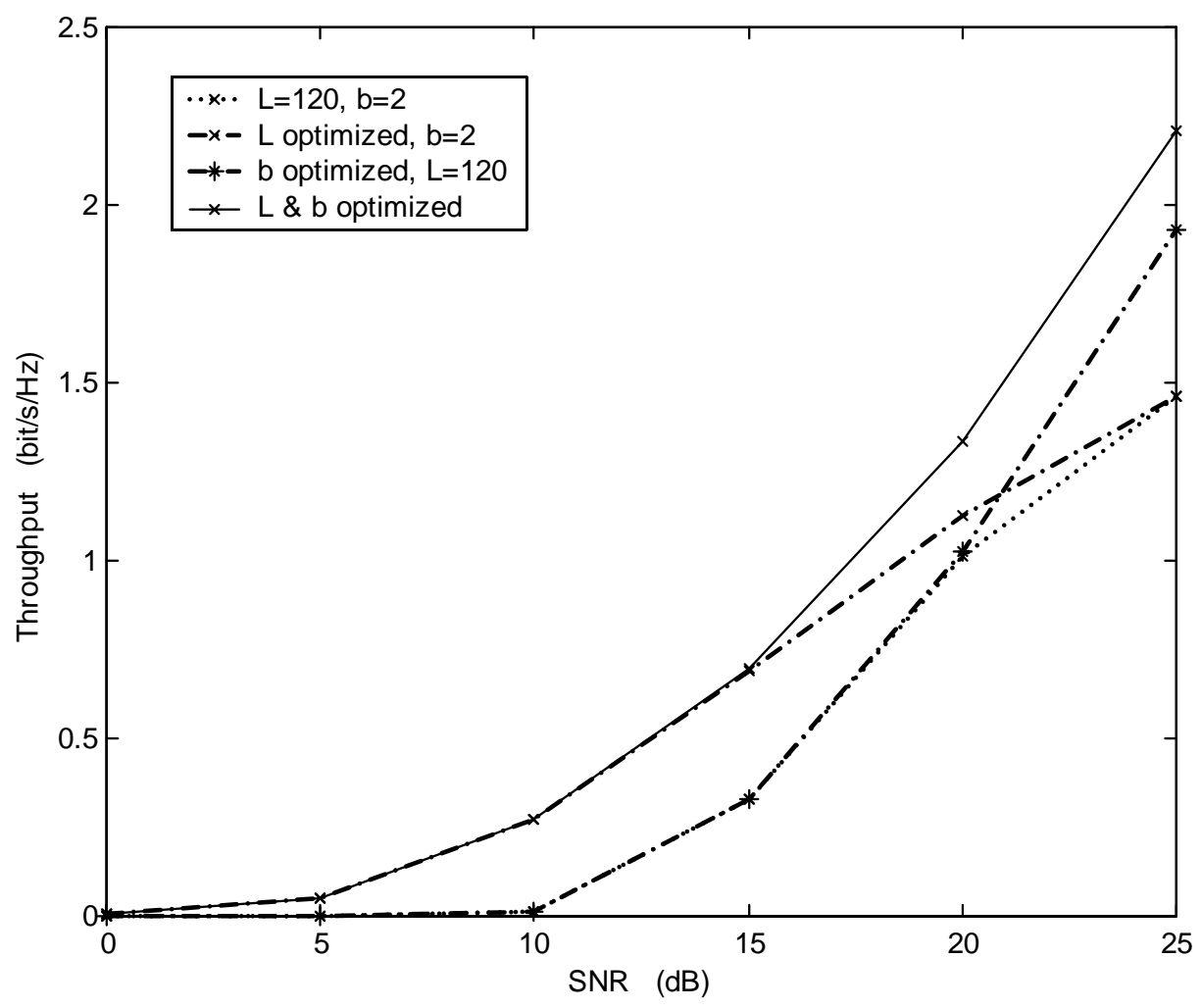

Fig. 9: Throughput optimization of the truncated ARQ scheme. 


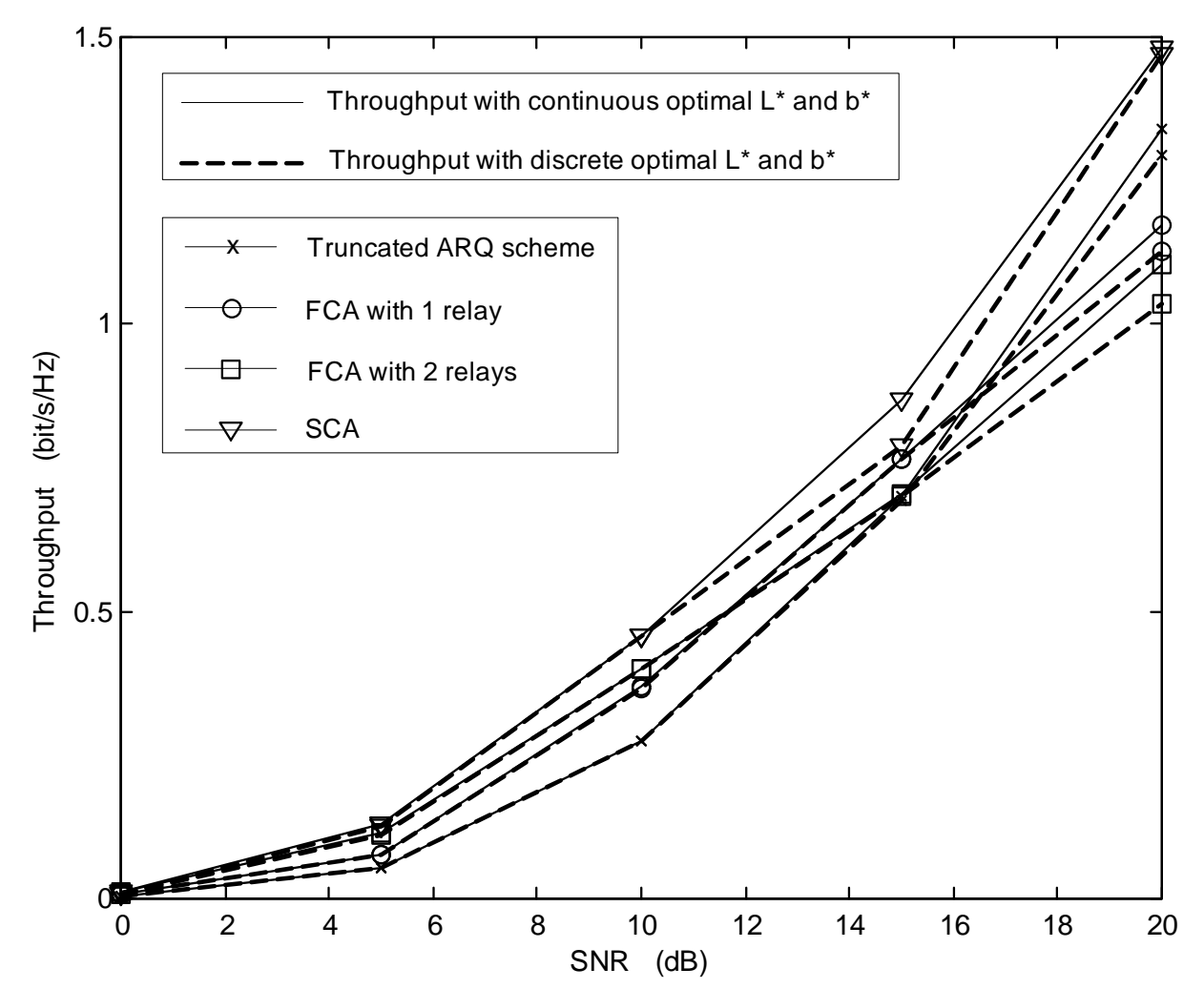

Fig. 10: Throughput with continuous optimal $L^{*}$ and $b^{*}$ and with discrete optimal $L^{*}$ and $b^{*}$ of the truncated ARQ scheme, FCA with 1 relay, FCA with 2 relays and SCA when $\gamma_{s s-r}=20 \mathrm{~dB}$. 